\title{
In situ evolution of the mechanical properties of stretchable and non-stretchable ePTFE vascular grafts and adjacent native vessels
}

\author{
Nele Famaey ${ }^{1}$, Jelle Verhoeven², Steven Jacobs², Matteo Pettinari², Bart Meyns ${ }^{2}$ \\ ${ }^{1}$ Biomechanics Section, Department of Mechanical Engineering, KU Leuven - Belgium \\ ${ }^{2}$ Department of Cardiac Surgery, University Hospital Gasthuisberg, KU Leuven - Belgium
}

\begin{abstract}
Introduction: The purpose of this study was to evaluate the evolution of the mechanical properties of stretchable and non-stretchable ePTFE vascular grafts over time following implantation, as well as those of the adjacent native vessels.

Methods: One stretchable and one non-stretchable graft were implanted in either carotid position of six sheep. After twelve weeks, the samples, as well as the distal adjacent native vessel, were explanted and evaluated mechanically by means of uniaxial tensile tests. These tests were performed in the axial as well as in the circumferential direction. Also, patches of these grafts were implanted subcutaneously in the abdominal wall of the same animals. At 2, 4, 6, and 8 weeks, patches were explanted and evaluated mechanically. Baseline mechanical tensile tests were also performed on non-implanted grafts and on native sheep carotid arteries.

Results: Statistical analysis shows a significant effect of implantation time on the tensile mechanical properties of ePTFE vascular grafts. This effect is present for stretchable as well as non-stretchable grafts, in axial as well as in circumferential direction of the material. Also the adjacent native vessels have a tendency to lose mechanical stiffness and strength in the circumferential direction, an effect which is most pronounced for stretchable grafts.

Conclusions: The mechanical properties of ePTFE vascular grafts and the adjacent native vessels significantly change as a function of implantation time. Compared to the native vessels, the grafts are still significantly stronger and stiffer, though longer term experiments are needed to evaluate whether and how much the decline would continue in time.
\end{abstract}

Keywords: ePTFE vascular grafts, Mechanical testing, Compliance

Accepted: October 22, 2014

\section{INTRODUCTION}

Vascular grafts are often required to enable revascularization in the case of cardiovascular disease. Though preferred, autologous venous grafts cannot always be harvested, cryografts are equally difficult to obtain (1) and tissue-engineered or gradually dissolving grafts are still in a developmental stage (2-4). This is why synthetic vascular grafts are regularly used, especially for larger vessels (>6 $\mathrm{mm}$ in diameter) (5).

When evaluating patency rates, Dacron and expanded polytetrafluoroethylene (ePTFE) are currently accepted as the most reliable synthetic vascular grafts, with no evidence for an advantage of one over the other (6). This study focuses on ePTFE grafts, specifically stretchable and non-stretchable Gore-Tex (Newark, DE, USA). ePTFE 
is a woven form of PTFE after a process of extrusion, rolling, stretching, and heating, such that a flexible, meshlike structure is created $(7,8)$. Both the stretchable and the non-stretchable grafts are made of ePTFE, but in the stretchable version the microfibrillar structure was crimped, allowing for a more supple graft with longitudinal distensibility (9).

Still, apart from the fact that current synthetic grafts do not mimic the antithrombogenic properties of native blood vessels (5), a major drawback is the high compliance mismatch between the graft and the native vessel, a fact which has been proven to play a role in patency and overall performance (10-12). The high stiffness of the grafts compared to native vessels is the result of a trade-off between compliance and mechanical stability, as attempts to increase graft compliance have not succeeded due to severe dilatation and failure (13).

However, although Dacron and ePTFE are considered biologically stable, even in these grafts dilatation is commonly reported, probably due to the degenerative effect of tissue fluids and enzymes (14). Specifically for ePTFE, Schröder et al (15) investigated over 100 patients with ePTFE grafts with both computerized tomography (CT) and ultrasound (US), and noted a significant dilatation in graft diameter (12.5\%), especially in the first three months after implantation. Also Doble et al reported a $50 \%$ decrease in tensile strength and $61 \%$ decrease in total elongation of ePTFE grafts explanted after an average of 74 weeks from children with congenital heart disease (16). Though rare, even ruptures do occur with ePTFE grafts (17).

The studies on ePTFE dilatation and rupture cited above are all clinical follow-ups and lack a well-controlled and properly quantified evaluation of the graft material properties as a function of implantation time. For example in the study of Doble et al (16), all samples had different implantation times and it is unclear whether the results of the mechanical tests pertain to the axial or the circumferential direction of the tissue, and whether stretchable or non-stretchable grafts were used. The primary purpose of the current study is therefore to quantitatively evaluate the evolution of the mechanical properties of ePTFE vascular grafts, both stretchable and non-stretchable, over time following implantation in sheep carotid position. Secondly, the effect of the compliance mismatch on the mechanical properties of the adjacent native vessels is evaluated for both graft types.

\section{MATERIALS AND METHODS}

\section{Animal experiments}

Six female Lovenaar sheep with a mean weight of $45 \mathrm{~kg}$ were selected and cared for in accordance with the 'Guide for the Care and Use of Laboratory Animals' (NIH publication 85-23, revised 1985). The study was approved by the local Ethics Committee. All sheep were pre-sedated with $4 \mathrm{mg} / \mathrm{kg}$ intravenous ketamin hydrochloride (Anesketin; Dechra, Shrewsbury, UK). General anesthesia was induced using 5\% isoflurane (Iso-Vet; Dechra, Shrewsbury, UK) until disappearance of the corneal reflex. Endotracheal intubation was performed and general anesthesia was maintained with a 98/2\% oxygen-isoflurane mixture. Arterial blood pressure, heart-rate, $\mathrm{ETCO}_{2}$, and $\mathrm{SpO}_{2}$ were continuously monitored. Both native carotid arteries were resected and replaced by a Gore-Tex stretchable graft on one side (catalogue no. S0604), and a non-stretchable graft (catalogue no. V06030L) on the other, both $6 \mathrm{~mm}$ in diameter. Next, four stretchable and four non-stretchable patches of GoreTex material, size $25 \times 25 \mathrm{~mm}$ were implanted subcutaneously at the thorax, halfway in between the vertebrae and the sternum.

After administration of $2 \mathrm{mg} / \mathrm{kg}$ intravenous meloxicam (Metacam; Boehringer Ingelheim, Ingelheim am Rhein, Germany), anesthesia was terminated by switching to $100 \%$ oxygen and the trachea tube was removed when spontaneous breathing of the animals resumed. Sheep were then housed at the university's animal facility with free roaming in an outside pen and ad libitum access to food and water.

At 2, 4, 6, and 8 weeks following surgery, one stretchable and one non-stretchable patch were removed. After 12 weeks, the carotid grafts and adjacent distal native vessels were removed and the animal was humanely sacrificed. All procedures were performed under general anesthesia as previously described and all samples were preserved in a sterile $0.9 \% \mathrm{NaCl}$ solution. Figure 1 shows examples of the explanted samples.

\section{Mechanical testing}

Before $24 \mathrm{~h}$ after explantation, each patch, interposition graft, and native vessel was cut into four rectangular segments of $10 \mathrm{~mm} \times 5 \mathrm{~mm}$. Two were oriented in the circumferential direction, two in the axial direction. These samples were mounted on a uniaxial tensile test bench 

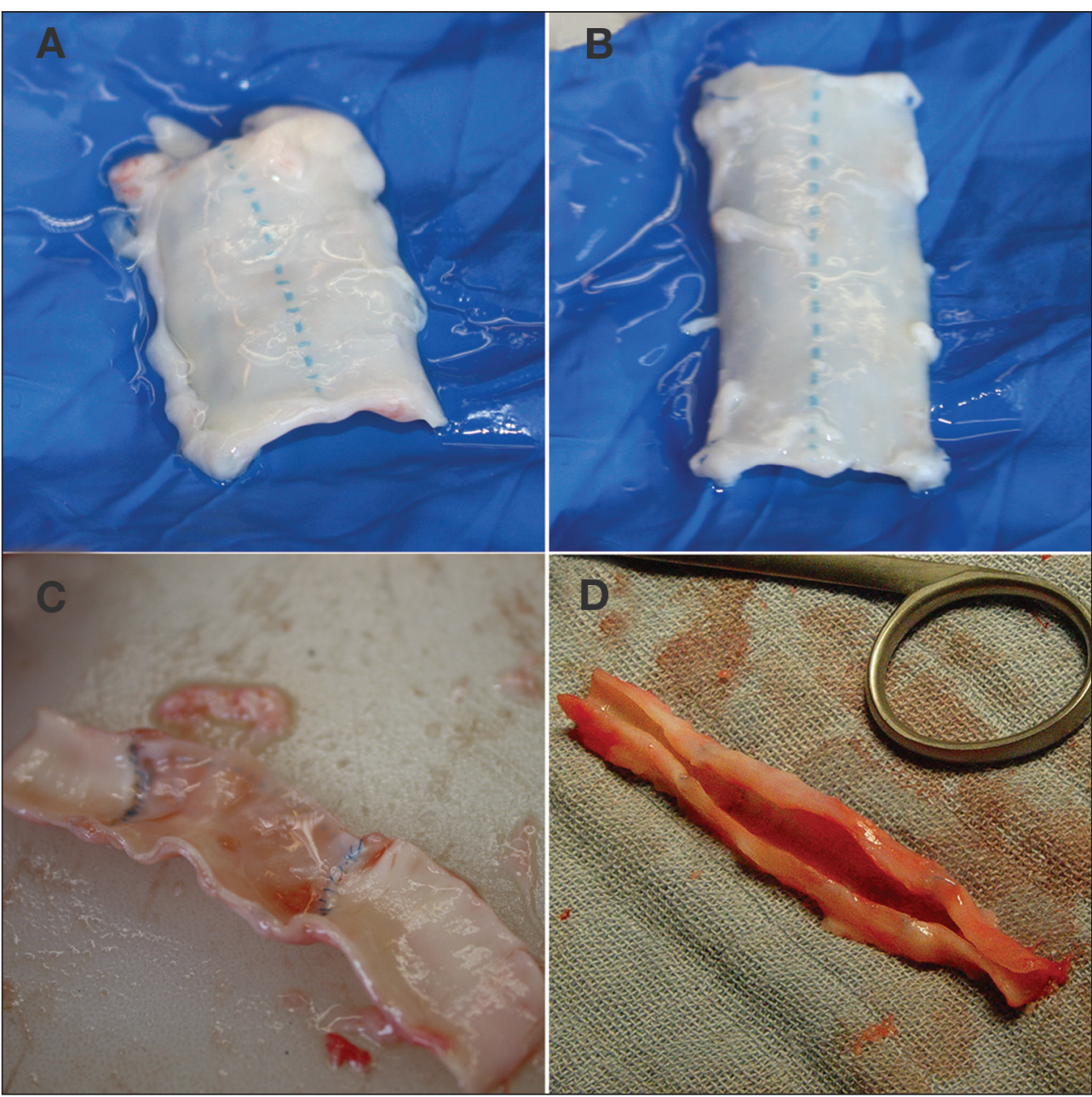

Fig. 1 - Examples of the explanted samples. (A) Subcutaneously implanted patches of stretchable Gore-Tex; and (B) non-stretchable Gore-Tex. Interposition grafts of Gore-Tex (C) stretchable and (D) non-stretchable material along with the adjacent native vessel.

(Instron 5567; Canton, MA, USA) and elongated to 50\% extension at a crosshead speed of $1 \mathrm{~mm} / \mathrm{s}$ and held at this level for $30 \mathrm{~s}$. Next, the samples were elongated until rupture.

The tests were performed with continuous recording (at $10 \mathrm{~Hz})$ of tensile force $\left(F^{\prime}\right)$ with a $1 \mathrm{kN}$ Instron load cell. Gauge length (I') was recorded with a Limess (Krefeld, Germany) optical measurement system (at $2.5 \mathrm{~Hz}$ ). The initial dimensions of each sample, namely thickness $\left(t^{\circ}\right)$ and width $\left(w^{0}\right)$, were measured with a digital caliper. The acquired data were processed to obtain the true stress $\left(\sigma_{1}^{\prime}\right)$ and the true strain $\left(\sigma_{1}\right)$ at each time point $i$ along the testing direction, according to the following formulae:

$$
\begin{aligned}
\sigma_{1}^{i} & =\frac{F^{i}}{A^{i}}, \\
\varepsilon_{1}^{i} & =\log \frac{l^{i}-l^{\circ}}{l^{\circ}}+1,
\end{aligned}
$$

with $A^{i}=\frac{W^{\circ} \cdot t^{\circ} \cdot l^{\circ}}{l^{i}}$ the cross-sectional area of the sample at a certain time point $i$, which is estimated based on the assumption of incompressibility ( $w=$ width, $t=$ thickness and $I=$ length).

Different parameters were extracted from each experimental data set using Matlab (Mathworks, Natick, MA, USA):

- $E_{10}, E_{20}$, etc., are the tangent moduli at $10 \%, 20 \%$, etc. strain, which are defined as the slope of the stressstrain curve at the respective strain level. These are measures of the stiffness of the material, by approximating it with a piecewise linear model. As with native arteries, Gore-tex material exhibits a nonlinear stiffening behavior, which is why the material stiffness cannot simply be characterized by one Young's modulus. For a discussion on nonlinearity please refer to section 4 . To interpret how these values relate to physiological loading levels, one can use equilibrium equations to calcu- 
late the circumferential stresses in a thick-walled tube as a function of its geometry and the luminal pressure. From the stress-strain curves, it can then be inferred which strain level and hence linearized stiffness lies in the physiological loading regime. For example, when a $6 \mathrm{~mm}$ diameter graft is implanted in a vessel under normal physiological pressures, the circumferential stress is around $0.13 \mathrm{MPa}$. Hence, parameter $E_{10}$ will most accurately describe its behaviour, as the tissue is hardly stretched due to its high stiffness. For native arteries on the other hand, parameters $E_{30}$ through $E_{60}$ are relevant at physiological loading levels, as the compliant tissue is stretched quite extensively in vivo, not only due to the physiological pressure levels, but also due to inherent residual stresses present in an artery.

- $\tau_{r}$ is the characteristic relaxation time during the 'hold'phase of the tensile test, defined as the ratio of the viscosity $\eta$ to the elasticity $E$ :

$$
\tau_{r}=\frac{\eta}{E}=-\frac{{ }^{t} \text { hold }}{\log \frac{\sigma^{e}}{\sigma^{b}}}
$$

with $t_{\text {hold }}=30$ s, i.e. the time at which the crosshead remained in place, and $\sigma_{1}^{b}$ and $\sigma_{1}{ }^{e}$ the stress at the beginning and at the end of the 'hold'-phase, respectively. This is a measure of the viscoelasticity of the material, and can be found by approximating the behavior with a Maxwell model, i.e., a series of a viscous damper and an elastic spring, which relates the strain rate - equal to zero in the 'hold'-phase - to the stress and stress rate at time $i$ as follows:

$$
0=E \dot{\varepsilon}_{1}^{i}=\dot{\sigma}_{1}^{i}+\frac{\sigma_{1}^{i}}{\tau_{r}}
$$

The nature of this model dictates that theoretically, the amount of relaxation time allowed will not affect the obtained value. From an experimental point of view however, more reliable results will be obtained for a longer relaxation time, as the tissue approaches its steady state, which will reduce the measurement error. Based on previous trials on similar material, $30 \mathrm{~s}$ relaxation time was chosen as an acceptable trade-off.

- $\quad \sigma_{1}^{\text {fail }}$ is the stress level at which failure of the sample occurred. This is a measure of the strength of the tissue.

Figure 2 provides a graphical overview of the estimated pa-

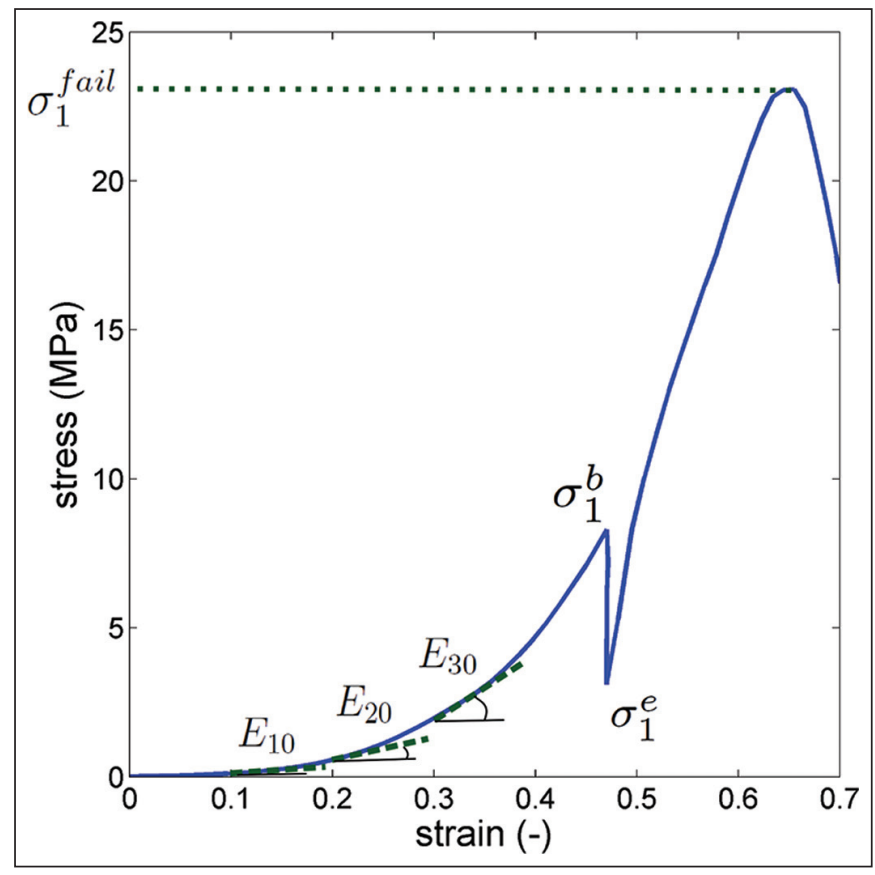

Fig. 2 - Overview of the different parameters extracted from the mechanical test.

rameters. The same tests were performed and parameters obtained for non-implanted material, with $n=8$ samples per combination of type (stretchable vs. non stretchable) and orientation (axial vs. circumferential). This data serves as the baseline for the longitudinal analysis. Also, tests were performed and parameters obtained for the native sheep carotid arteries that were removed from each of the sheep, with $n=12$ samples per orientation.

\section{Statistical analysis}

Statistical analysis was performed in SPSS (IBM SPSS Statistics for Windows, Version 21, Armond, NY, USA), for which three datasets were considered. The first dataset contains the longitudinal evolution of the aforementioned parameters for the implanted patches. Repeated measurements were taken at 5 time points $(0,2,4,6$ and 8 weeks), for the stretchable and the non-stretchable ePTFE, in the axial and circumferential direction. Two measurements were available for each combination of time, direction, type and sheep. At baseline, 8 measurements per combination of direction and type were available.

The second dataset contains the measurements of the graft interpositions, implanted in the carotid position for 12 


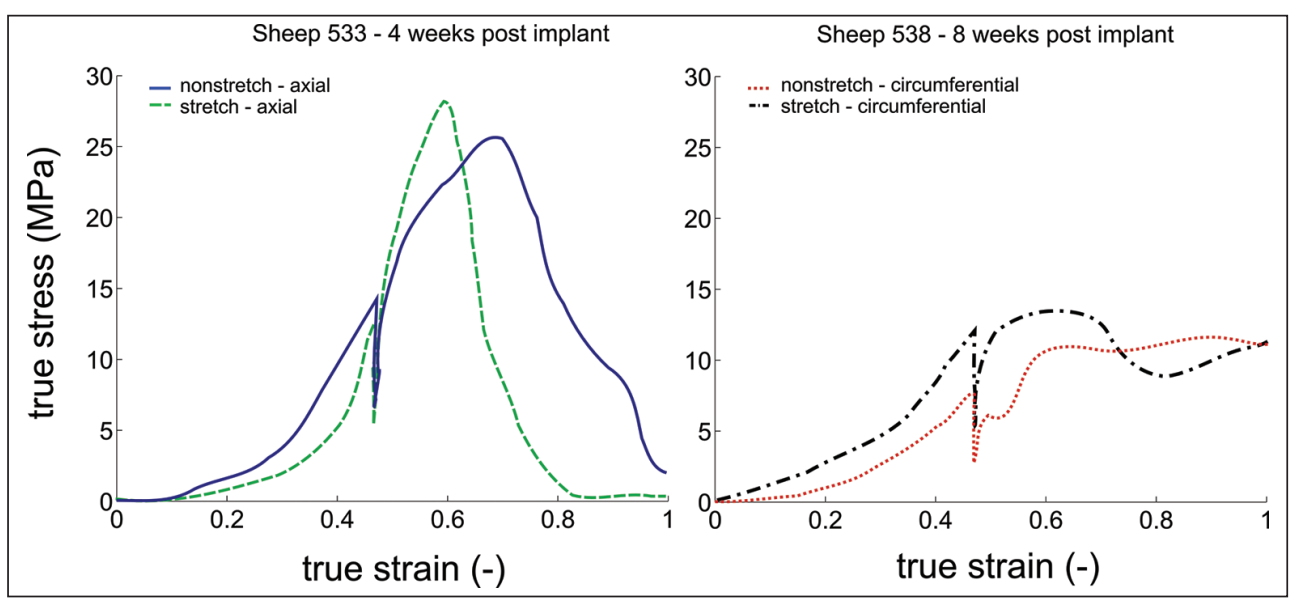

Fig. 3 - Examples of stress-strain curves obtained through mechanical testing. The left image shows axial stress-strain curves for a stretchable and a non-stretchable sample 4 weeks post-implant in the same animal. The right image shows circumferential stress-strain curves for both tissue types 8 weeks post-implant in another sheep.

weeks. Again, two measurements were available for each combination of direction, type, and sheep. These were compared to the same baseline measurements as in dataset 1 . To account for the nonlinearity of the response variables, the following transformations were performed for both datasets: $\log E_{i}=10 \log \left(E_{i}+1\right)$ (with $\left.i=10,20,30\right), \log \sigma_{1}^{\text {fail }}=100$

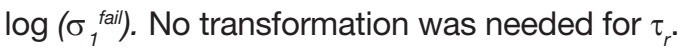

To assess the effect of time on the grafts (0-2-4-6-8 weeks for dataset 1, 0-12 weeks for dataset 2), mixed models were fitted on the data. The different combinations of type and direction (axial-stretchable, axial-non-stretchable, circumferential-stretchable, circumferential-non-stretchable) were considered as groups. For the response variables $\log E_{i}$, and $\tau_{r}$, a mixed model with random intercept was considered, with fixed effects for time, group, and the interaction effect time ${ }^{*}$ group. For the response variable $\log \sigma_{1}^{\text {fail }}$ of dataset 1, time $^{2}$ was also considered as a fixed effect. For a rationale on this statistical analysis method, please refer to the Appendix. The third dataset contains the measurements of the native sheep carotid arteries. Distinction was made between vessels adjacent to a non-stretchable and to a stretchable graft. Again, two measurements were available for each combination of direction, type, and sheep. These were compared to the properties of control native sheep carotid arteries. For this dataset, paired data was not available. Therefore, two-sample $t$-tests assuming non-equal variances were performed.

Effects were considered significant if $p<0.05$. When multiple groups were compared, a Bonferroni correction was made.

\section{RESULTS}

Figure 3 shows a number of representative stress-strain curves obtained through mechanical testing. Tables I and II show the average values and standard deviations for the mechanical parameters described in section 2, for each combination of time, direction and type, averaged over the number of sheep. Also, the values of a native carotid artery are shown. Table III shows the values of the slopes over time of the estimated marginal regression lines, found for each mechanical parameter for each combination of type and direction, along with the confidence values. The values are displayed for the evolution of the subcutaneous patches from baseline to 8 weeks, as well as for the evolution of the carotid grafts from baseline to 12 weeks.

Statistical analysis shows a significant effect of time for the parameters $\log E_{i}$, for each combination of type and direction. The effect is present for the subcutaneous patches as well as for the carotid grafts. The effect was positive for stretchable grafts in axial direction and negative for the other three combinations. This means that the stiffness of the stretchable grafts increases in the axial direction (in other words, they lose their stretchability), and decreases in the other direction. The stiffness also decreases for the non-stretchable grafts (in other words, they become more compliant).

A significant effect of time can be observed for the relaxation time $\tau_{r}$, for each combination of type and direction. The effect is present for the subcutaneous patches as well as for the carotid grafts. In all cases, the effect is positive, meaning the relaxation time increases with time. In other words, the grafts become less viscoelastic as a function 
TABLE I - AXIAL DIRECTION AVERAGE VALUES AND STANDARD DEVIATION

\begin{tabular}{|c|c|c|c|c|c|c|c|c|c|c|}
\hline & \multicolumn{2}{|c|}{$\sigma_{1}^{\text {fail }}$ [MPa] } & \multicolumn{2}{|c|}{$E_{10}[\mathrm{MPa}]$} & \multicolumn{2}{|c|}{$E_{20}[\mathrm{MPa}]$} & \multicolumn{2}{|c|}{$E_{30}[\mathrm{MPa}]$} & \multicolumn{2}{|c|}{$\tau_{r}[\mathbf{s}]$} \\
\hline & str & non & str & non & str & non & str & non & str & non \\
\hline \multirow[t]{2}{*}{0} & 41.48 & 32.40 & 0.77 & 26.62 & 1.33 & 136.67 & 2.70 & 158.25 & 44.26 & 51.34 \\
\hline & \pm 3.34 & \pm 3.80 & \pm 0.47 & \pm 25.53 & \pm 0.70 & \pm 39.52 & \pm 1.36 & \pm 53.68 & \pm 4.33 & \pm 2.95 \\
\hline \multirow[t]{2}{*}{2} & 33.03 & 24.18 & 1.88 & 21.05 & 11.17 & 104.50 & 36.57 & 103.08 & 56.56 & 60.30 \\
\hline & \pm 11.80 & \pm 11.38 & \pm 1.07 & \pm 29.56 & \pm 13.86 & \pm 66.24 & \pm 45.59 & \pm 70.42 & \pm 7.39 & \pm 14.64 \\
\hline \multirow[t]{2}{*}{4} & 29.64 & 22.78 & 2.17 & 8.29 & 5.61 & 37.65 & 9.40 & 54.53 & 52.67 & 61.95 \\
\hline & \pm 9.73 & \pm 9.51 & \pm 0.27 & \pm 6.02 & \pm 1.36 & \pm 49.10 & \pm 4.71 & \pm 56.34 & \pm 9.01 & \pm 16.58 \\
\hline \multirow[t]{2}{*}{6} & 26.57 & 14.90 & 2.07 & 8.68 & 5.45 & 20.22 & 4.47 & 56.98 & 57.25 & 54.87 \\
\hline & \pm 13.30 & \pm 3.52 & \pm 1.54 & \pm 3.46 & \pm 4.77 & \pm 11.23 & \pm 3.41 & \pm 59.90 & \pm 10.93 & \pm 26.49 \\
\hline \multirow[t]{2}{*}{8} & 35.15 & 20.50 & 3.51 & 10.25 & 5.56 & 29.34 & 6.06 & 53.22 & 61.65 & 67.62 \\
\hline & \pm 12.94 & \pm 11.73 & \pm 3.05 & \pm 4.95 & \pm 4.29 & \pm 18.91 & \pm 3.91 & \pm 29.88 & \pm 13.86 & \pm 7.02 \\
\hline \multirow[t]{2}{*}{12} & 21.29 & 19.46 & 7.21 & 22.31 & 21.77 & 65.96 & 30.13 & 77.15 & 69.02 & 66.68 \\
\hline & \pm 8.03 & \pm 7.68 & \pm 9.25 & \pm 22.48 & \pm 21.44 & \pm 48.92 & \pm 16.26 & \pm 51.24 & \pm 6.04 & \pm 15.49 \\
\hline \multirow[t]{2}{*}{ Carotid } & 5.30 & & 0.21 & & 0.54 & & 0.96 & & 115.87 & \\
\hline & \pm 1.96 & & \pm 0.16 & & \pm 0.64 & & \pm 0.79 & & \pm 70.21 & \\
\hline
\end{tabular}

Average values and standard deviations for the mechanical parameters in axial direction for the stretchable (str) and non-stretchable (non) grafts at different weeks after implantation and for the native sheep carotid artery.

TABLE II - CIRCUMFERENTIAL DIRECTION AVERAGE VALUES AND STANDARD DEVIATIONS

\begin{tabular}{|c|c|c|c|c|c|c|c|c|c|c|}
\hline & \multicolumn{2}{|c|}{$\sigma_{1}^{\text {fail }}[\mathrm{MPa}]$} & \multicolumn{2}{|c|}{$E_{10}[\mathrm{MPa}]$} & \multicolumn{2}{|c|}{$E_{20}[\mathrm{MPa}]$} & \multicolumn{2}{|c|}{$E_{30}[\mathrm{MPa}]$} & \multicolumn{2}{|c|}{$\tau_{r}[\mathbf{s}]$} \\
\hline & str & non & str & non & str & non & str & non & str & non \\
\hline \multirow[t]{2}{*}{0} & 20.59 & 10.12 & 20.57 & 10.10 & 69.25 & 45.98 & 83.89 & 68.98 & 51.02 & 62.69 \\
\hline & \pm 3.38 & \pm 1.54 & \pm 3.17 & \pm 0.88 & \pm 23.39 & \pm 21.99 & \pm 13.95 & \pm 28.52 & \pm 5.17 & \pm 4.77 \\
\hline \multirow[t]{2}{*}{2} & 22.67 & 10.91 & 22.27 & 10.72 & 34.85 & 18.89 & 97.23 & 62.53 & 62.82 & 70.50 \\
\hline & \pm 20.55 & \pm 3.22 & \pm 25.95 & \pm 3.25 & \pm 43.77 & \pm 8.00 & \pm 130.52 & \pm 26.11 & \pm 8.80 & \pm 8.26 \\
\hline \multirow[t]{2}{*}{4} & 13.91 & 9.64 & 11.30 & 9.44 & 18.19 & 15.68 & 41.52 & 21.19 & 68.11 & 70.94 \\
\hline & \pm 4.96 & \pm 4.30 & \pm 5.92 & \pm 3.26 & \pm 11.50 & \pm 13.60 & \pm 39.88 & \pm 12.85 & \pm 7.87 & \pm 5.34 \\
\hline \multirow[t]{2}{*}{6} & 13.97 & 8.90 & 15.61 & 14.87 & 16.61 & 24.96 & 42.74 & 29.10 & 63.67 & 67.47 \\
\hline & \pm 4.08 & \pm 3.31 & \pm 4.95 & \pm 4.74 & \pm 5.84 & \pm 11.17 & \pm 32.14 & \pm 22.84 & \pm 2.88 & \pm 3.69 \\
\hline \multirow[t]{2}{*}{8} & 16.88 & 9.49 & 17.01 & 10.27 & 26.61 & 23.84 & 39.60 & 21.99 & 64.56 & 69.51 \\
\hline & \pm 5.83 & \pm 2.65 & \pm 3.99 & \pm 6.10 & \pm 8.61 & \pm 10.30 & \pm 21.63 & \pm 14.34 & \pm 2.96 & \pm 10.13 \\
\hline \multirow[t]{2}{*}{12} & 13.79 & 10.62 & 8.78 & 6.84 & 18.16 & 11.62 & 40.17 & 28.65 & 70.12 & 77.14 \\
\hline & \pm 4.26 & \pm 1.95 & \pm 2.73 & \pm 3.69 & \pm 13.32 & \pm 3.96 & \pm 12.18 & \pm 14.14 & \pm 10.22 & \pm 7.13 \\
\hline \multirow[t]{2}{*}{ Carotid } & 3.86 & & 0.49 & & 0.94 & & 1.52 & & 73.75 & \\
\hline & \pm 1.84 & & \pm 0.25 & & \pm 0.69 & & \pm 1.34 & & \pm 26.10 & \\
\hline
\end{tabular}

Average values and standard deviations for the mechanical parameters in circumferential direction for the stretchable (str) and non-stretchable (non) grafts at different weeks after implantation and for the native sheep carotid artery. 
TABLE III - ESTIMATED MARGINAL REGRESSION LINES

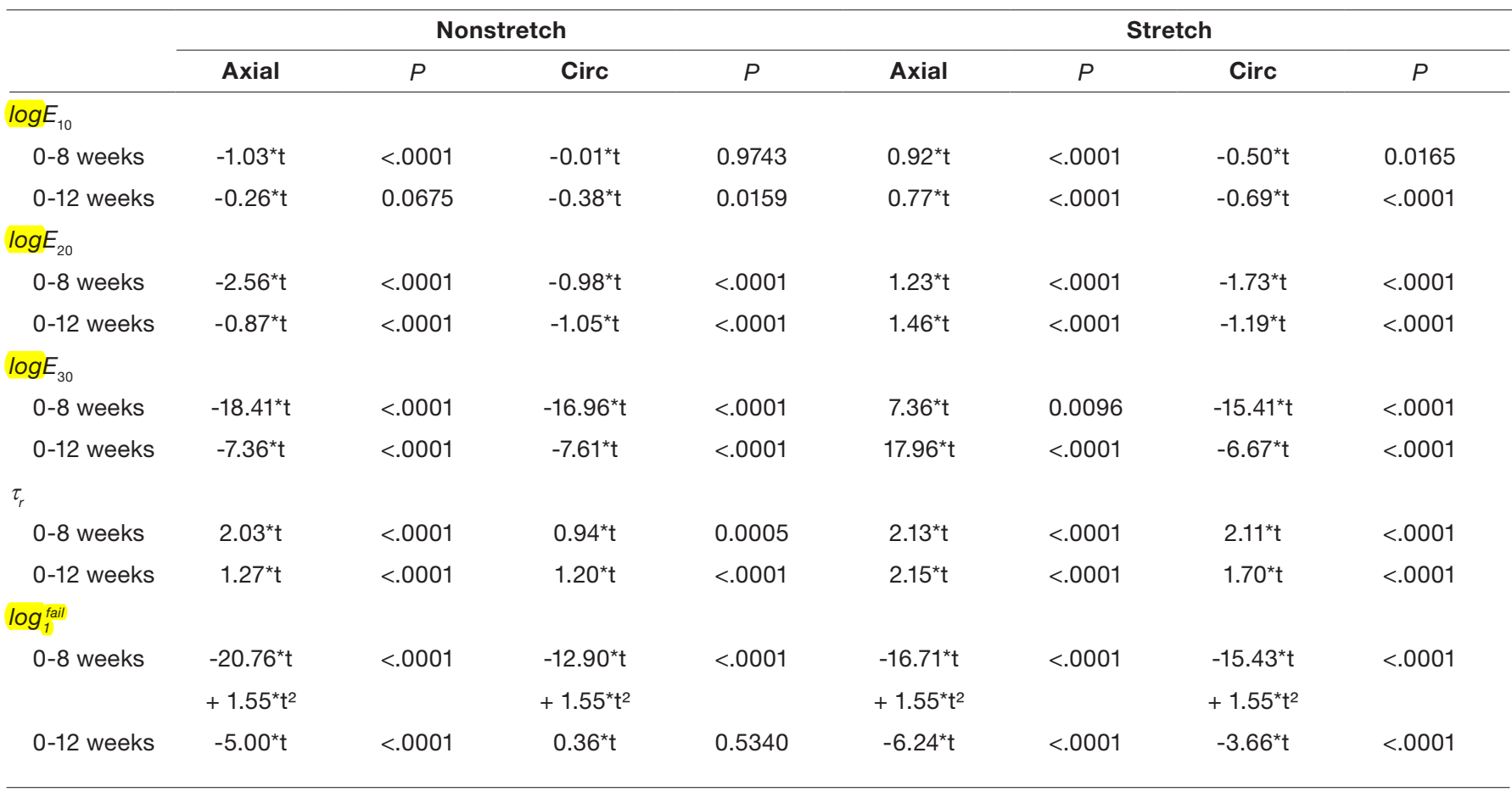

Values of the slopes of the estimated marginal regression lines, for $\log E_{10}, \log E_{20}, \log E_{30}, \tau_{r}$ and $\log$ fail, for each combination of type and direction, along with the confidence values. The unit of time is the number of weeks. [AU: Please provide significance of symbol [*]

TABLE IV - CAROTID ARTERIES AVERAGE VALUES AND STANDARD DEVIATIONS

\begin{tabular}{|c|c|c|c|c|c|c|c|}
\hline & & $\sigma_{1}^{\text {fail }}[\mathrm{MPa}]$ & $E_{30}[\mathrm{MPa}]$ & $E_{40}[\mathrm{MPa}]$ & $E_{50}[\mathrm{MPa}]$ & $E_{60}[\mathrm{MPa}]$ & $\tau_{r}[\mathbf{s}]$ \\
\hline \multirow[t]{8}{*}{ circ } & carotis_non & 4.44 & 1.24 & 3.17 & 6.60 & 3.20 & 60.57 \\
\hline & & \pm 2.00 & \pm 0.53 & \pm 1.85 & \pm 3.75 & \pm 1.57 & \pm 8.22 \\
\hline & $P$ & 0.48 & $0.04^{\star *}$ & 0.18 & 0.14 & $0.03^{\star *}$ & 0.08 \\
\hline & carotis_str & 3.02 & 1.48 & 1.64 & 4.48 & 4.20 & 84.08 \\
\hline & & \pm 1.69 & \pm 1.54 & \pm 1.02 & \pm 2.88 & \pm 1.60 & \pm 35.08 \\
\hline & $P$ & $0.04^{\star *}$ & $0.10^{\star}$ & $0.04^{* *}$ & $0.08^{*}$ & $0.04^{\star *}$ & 0.14 \\
\hline & carotis & 4.48 & 2.64 & 4.89 & 13.58 & 16.34 & 77.17 \\
\hline & & \pm 0.88 & \pm 1.85 & \pm 4.60 & \pm 16.74 & \pm 12.82 & \pm 0.41 \\
\hline \multirow[t]{8}{*}{ axial } & carotis_non & 5.82 & 1.40 & 7.76 & 18.72 & 12.58 & 108.66 \\
\hline & & \pm 1.80 & \pm 0.96 & \pm 8.09 & \pm 20.24 & \pm 10.95 & \pm 78.97 \\
\hline & $P$ & 0.43 & 0.16 & $0.03^{* *}$ & $0.08^{*}$ & 0.29 & 0.14 \\
\hline & carotis_str & 4.31 & 0.66 & 2.13 & 6.66 & 11.25 & 127.54 \\
\hline & & \pm 1.93 & \pm 0.35 & \pm 2.04 & \pm 5.33 & \pm 5.64 & \pm 76.06 \\
\hline & $P$ & $0.09^{*}$ & 0.11 & 0.30 & 0.42 & 0.21 & 0.26 \\
\hline & carotis & 5.66 & 1.00 & 1.70 & 7.35 & 16.50 & 150.12 \\
\hline & & \pm 2.28 & \pm 0.76 & \pm 0.83 & \pm 7.16 & \pm 13.68 & \pm 56.07 \\
\hline
\end{tabular}

Average values and standard deviations for the mechanical parameters of the carotid arteries, in control condition (carotis) and after 12 weeks adjacent to a stretchable (carotis_str) or a non-stretchable (carotis_non) graft. $P$-values indicating significant or marginally significant differences from the control values are marked with ${ }^{* *}$ and *, respectively. 
of time.

The stress value at which the material fails $\left(\sigma_{1}{ }^{\text {fail }}\right)$ decreases significantly over time for the stretchable grafts and for the non-stretchable grafts in the axial direction. The effect is present for the subcutaneous patches as well as for the carotid grafts. In other words, these grafts lose their axial tensile strength over time. For the non-stretchable grafts in the circumferential direction, the effect is less pronounced for the subcutaneous patches and is unnoticeable for the graft implants. Note that the strength of the non-stretchable grafts in circumferential direction is already low to begin with compared to the other groups at baseline.

Tables I and II also show how a native sheep carotid artery is still far weaker than any of the grafts in any direction, even after 8 or 12 weeks of implantation. The stiffness of the native artery is also significantly lower than that of the grafts. At baseline, only the stretchable graft comes close to the native properties in the axial direction, which makes these stretchable grafts easier to implant. After implantation, the stretchable graft loses its stretchability and again becomes far stiffer than the native carotid. In the circumferential direction, grafts have similar viscoelastic properties to native sheep carotid arteries after implantation. In the axial direction, native carotid arteries seem less viscoelastic than either of the grafts, though this result is not statistically significant.

Table IV shows the mechanical properties of the native vessels that were adjacent to the implanted grafts after 12 weeks. Here, one can see that in the axial direction, the properties hardly seem to change compared to the control vessels, regardless of the type of graft. In the circumferential direction, the results indicate that vessels adjacent to an ePTFE graft lose their stiffness and their strength, regardless of the type of graft, but the effect is slightly more pronounced in the case of a stretchable graft.

\section{DISCUSSION}

The first conclusion that can be drawn from the results is that ePTFE grafts show a significant weakening after implantation $\left(\sigma_{1}^{\text {fail }}\right)$, in the axial direction for both types and in the circumferential direction for the stretchable grafts. These results seem to confirm the clinical ndings of Doble et al (16). However, the tests described in the current study were performed in a controlled manner and along two directions. Though Schröder et al (15) also found that dilatation was most pronounced in the first three months, longer-term controlled experiments as described here are needed to determine whether and how much the graft properties would further decline as a function of time and how, this could increase the potential risk of aneurysm formation and failure as a clinical consequence.

A second conclusion is that the stiffness of both grafts also decreases with implantation time, except for the stretchable graft in the axial direction, which significantly increases. The stiffening of the stretchable graft in the axial direction causes the relatively close, initial match in properties with the native artery in this direction to disappear. For the circumferential direction of the stretchable graft and for the non-stretchable graft, the stiffness values also still largely exceed that of the native sheep carotid artery after 12 weeks, i.e., the initial compliance mismatch slightly diminishes, but still remains. Note also that the circumferential stiffness of stretchable grafts is higher than that of the non-stretchable grafts. This effect is already present at baseline, indicating that the difference is induced during the fabrication process enabling stretchability in the axial direction, namely the crimping process which compresses the microfibrils. The actual micromechanical cause of this effect and whether and how this initial bias plays a role in the in situ behavior of both grafts would be an interesting topic to investigate further, but falls beyond the scope of this study, which empirically discusses the evolution of the mechanical properties of these grafts.

Thirdly, one can conclude that the circumferential properties of the native arteries adjacent to the implanted grafts are negatively affected over time. This might be attributed to functional adaptation as a consequence of the compliance mismatch between the native vessel and the graft. These discontinuities in compliance cause critical changes in local hemodynamics, altering the local pressure and velocity gradients (18). As was also clear from Table II, the stretchable grafts are actually stiffer in the circumferential direction compared to the nonstretchable grafts. This higher compliance mismatch also seems to lead to a stronger decline of the mechanical properties of the adjacent vessels. The axial properties of the native vessels remain largely unaffected, as a compliance mismatch will have only a limited influence on the axial loading profile, which is already quite low to begin with.

Summarizing the above findings, one can see that all grafts 
lose some stiffness and strength in the course of 12 weeks; only the axial direction of the stretchable graft stiffens up to converge towards values of a non-stretchable graft. Also, a stretchable graft is actually stiffer and stronger than a non-stretchable graft in the circumferential direction, and despite the general decline of the mechanical properties of either graft, this difference remains present after 12 weeks. This stronger compliance mismatch with the native vessel seems to yield a slightly higher degradation of the mechanical properties of the adjacent vessels. Nevertheless, these differences are small and the clear benefit of stretchable grafts is their ease of implantation.

Note that both native arteries and ePTFE material exhibit anisotropic, nonlinear stiffening behavior, which is why the material stiffness cannot simply be characterized by one Young's modulus. Characterizing the material in a piecewise, linear manner along each direction, as was done in this study, is of course an approximation, which is not ideal for advanced mathematical simulations. For these situations, advanced constitutive models such as the Holzapfel-Gasser-Ogden material model for cardiovascular tissue is preferred (19). However, this piecewise linear approach allows a straightforward interpretation of the experimental data, which is why it was chosen here.

This study shows the evolution of ePTFE grafts after implantation in subcutaneous position after 2, 4, 6, and 8 weeks, and in carotid position after 12 weeks. Despite the difference in location, the decline of mechanical properties that was noted followed the same trends and the values were in the same order of magnitude, indicating the suitability of the subcutaneous model. Still, care should be taken when comparing values from both implant positions.

\section{CONCLUSIONS}

The mechanical properties of ePTFE vascular grafts significantly change as a function of implantation time. Still, even after 12 weeks, a large compliance mismatch with the native vessels remains. Due to this mismatch, the circumferential mechanical properties of the adjacent native vessels will also slightly decline over time. While presenting the advantage of easier implantation, a stretchable ePTFE graft will lose its axial stretchability soon after implantation and yield even slightly stronger negative effects on the adjacent vessels compared to the non-stretchable version. This can be attributed to the higher compliance mismatch in the circumferential direction of these stretchable grafts. Compared to the native vessels, the grafts are still significantly stronger and stiffer, though longer-term experiments are needed to evaluate whether and how much the decline of the mechanical properties would continue in time, both for the grafts themselves and for the adjacent native vessels.

\section{APPENDIX}

Mixed effects models were used for the statistical analysis of the first and second dataset, an approach commonly used for longitudinal studies. In this approach, the response variable of a certain sample is considered to be the sum of a number of fixed and random effects. The response variables under consideration here are the mechanical parameters described above under Mechanical testing, which were transformed as presented in the Statistical analysis section to linearize the response of the variable.

In a longitudinal context, data are often clustered because a given subject is measured repeatedly over time. In this study, the sheep in which the graft tissue was implanted is measured several times. These data are then modeled by introducing a random sheep effect. Fixed effects are the primary interest in the current (and most) studies. In this study, time after implantation, the square of time, direction (circumferential or axial), and type of graft tissue (stretchable or non-stretchable) were considered as fixed effects. The latter two were combined into one effect, thereby distinguishing between four discrete groups.

A linear mixed model is now used to model the relationship between the response variable and the set of fixed and random effects. Per group, a certain response variable $Y_{i j}$, i.e., the $j$ th measurement of the $i$ th sheep taken at time $t_{j}$, where $Y$ represents one of the (transformed) mechanical parameters, e.g. $\log \sigma_{1}^{\text {fail }}$, can be modeled as:

$$
\begin{aligned}
& Y_{i j}=\beta_{1}+b_{i}+\beta_{2} t_{j}+\beta_{9} t_{j}^{2}+\varepsilon_{i j}, \text { if group } 1 \text { (nonstretch axial) } \\
& Y_{i j}=\beta_{3}+b_{i}+\beta_{4} t_{j}+\beta_{9} t_{j}^{2}+\varepsilon_{i j}, \text { if group } 2 \text { (nonstretch circ) } \\
& Y_{i j}=\beta_{5}+b_{i}+\beta_{6} t_{j}+\beta_{9} t_{j}^{2}+\varepsilon_{i j}, \text { if group } 3 \text { (stretch axial) } \\
& Y_{i j}=\beta_{7}+b_{i}+\beta_{8} t_{j}+\beta_{9} t_{j}^{2}+\varepsilon_{i j}, \text { if group } 4 \text { (stretch circ) }
\end{aligned}
$$


where $\beta_{1,3,5,7}$ represent the fixed intercept for each group, $b_{i}$ represents the random intercept caused by the sheep, $\beta_{2,4,6,8}$ represent the fixed effect of time for each group, $\beta_{9}$ represents the fixed effect of time $^{2}$ and $\varepsilon_{i j}$ contains the residuals.

In our study, interest goes out to [AUTHOR: I'm not sure what is intended by "interest goes out to". Kindly verify the meaning in English] the fixed effect of time, i.e., $\beta_{2,4,6,8}$ and $\beta_{9}$. Hence, after fitting the above model to our dataset, it is tested whether the null hypothesis, i.e., $H_{0}: \beta_{2}=\beta_{4}=\beta_{6}=\beta_{8}$ can be rejected. If this is the case, the effect of time is significantly different in the four groups. Moreover, we test within each group whether there is a significant effect of time by checking whether $H_{0}: \beta_{i}=0$ can be rejected for $i=2$, $4,6,8$. Finally, contrasts are formulated to see whether the slopes for specific groups (e.g. $H_{0}: \beta_{2}=\beta_{6}$ ) are significantly different from each other. Because multiple groups were compared, a Bonferroni correction was made. For more information on the mixed-models, the reader is referred to (20), for example.

\section{ACKNOWLEDGEMENTS}

The authors would like to thank the Leuven Statistics Research Centre, specifically Prof. An Carbonez and Dr. Anna Ivanova, for their help in the statistical analysis.

Financial Support: This work was supported by a PhD grant from the Institute for the Promotion of Innovation through Science and Technology in Flanders (I.W.T.-Vlaanderen) and by a postdoctoral fellowship from the Research Foundation- Flanders (FWO).

Conflict of Interest: No conflict of interest exists for any of the authors.

Address for correspondence:

Dr. Ir. Nele Famaey

Biomechanics Section

Celestijnenlaan 300C

B-3001 Heverlee KU Leuven, Belgium

nele.famaey@mech.kuleuven.be

\section{REFERENCES}

1. Bia D, Zócalo Y, Armentano RL, et al. Post-implant evaluation of the anastomotic mechanical and geometrical coupling between human native arteries and arterial cryografts implanted in lower-limb: mechanical, histological and ultraestructural studies of implanted cryografts. Cryobiology. 2012;64(1): 50-59.

2. Soldani G, Losi P, Bernabei M, et al. Long term performance of small-diameter vascular grafts made of a poly(ether)urethane-polydimethylsiloxane semi-interpenetrating polymeric network. Biomaterials. 2010;31(9):2592-2605.

3. Stickler P, De Visscher G, Mesure L, et al. Cyclically stretching developing tissue in vivo enhances mechanical strength and organization of vascular grafts. Acta Biomater. 2010;6(7):2448-2456.

4. Rémy M, Durand M, Menu P, et al. Interspecies differences with in vitro and in vivo models of vascular tissue engineering. Biomaterials. 2013;34(38):9842-9852.

5. Hoshi RA, Van Lith R, Jen MC, Allen JB, Lapidos KA, Ameer $G$. The blood and vascular cell compatibility of heparin-modified ePTFE vascular grafts. Biomaterials. 2013;34(1):30-41.

6. Takagi H, Goto S-N, Matsui M, Manabe H, Umemoto T. A contemporary meta-analysis of Dacron versus polytetrafluoroethylene grafts for femoropopliteal bypass grafting. J Vasc Surg. 2010;52(1):232-236.
7. Jørgensen CS, Paaske WP. Physical and mechanical properties of ePTFE stretch vascular grafts determined by timeresolved scanning acoustic microscopy. Eur J Vasc Endovasc Surg. 1998;15(5):416-422.

8. Hao X, Zhang J, Guo Y, Zhang H. Studies on Porous and Morphological Structures of Expanded PTFE Membrane Through Biaxial Stretching Technique. Int Nonwovens J. 2005;14(2): 31-38.

9. Erdoes LS, Berman SS, Bernhard VM, Mulcahy M, Hunter GC. Clinical and CT evaluation of a new stretch polytetrafluoroethylene aortic graft. Ann Vasc Surg. 1995;9(5):441-447.

10. Kidson IG. The effect of wall mechanical properties on patency of arterial grafts. Ann R Coll Surg Engl. 1983;65(1): 24-29.

11. Sarkar S, Salacinski HJ, Hamilton G, Seifalian AM. The mechanical properties of infrainguinal vascular bypass grafts: their role in influencing patency. Eur J Vasc Endovasc Surg. 2006;31(6):627-636.

12. Spadaccio C, Rainer A, Barbato R, Chello M, Meyns B. The fate of large-diameter Dacron ${ }^{\circledR}$ vascular grafts in surgical practice: are we really satisfied? Int J Cardiol. 2013;168(5): 5028-5029.

13. Xue L, Greisler HP. Biomaterials in the development and future of vascular grafts. J Vasc Surg. 2003;37(2):472-480.

14. Blumenberg R, Anderson J, Gelfand M, Skudder P, Bowers C. Histologic Evaluation of Dacron ${ }^{\circledR}$ and PTFE Graft Material 
Explanted From Humans After 4 to 20 Years In Vivo. Vasc Endovascular Surg. 2000;34(6):505-512.

15. Schroeder TV, Eldrup N, Just S, Hansen M, Nyhuus B, Sillesen $\mathrm{H}$. Dilatation of aortic grafts over time: what to expect and when to be concerned. Semin Vasc Surg. 2009;22(2):119-124.

16. Doble M, Makadia N, Pavithran S, Kumar RS. Analysis of explanted ePTFE cardiovascular grafts (modified BT shunt). Biomed Mater. 2008;3(3):034118.

17. Shibutani S, Obara H, Kakefuda T, Kitagawa Y. Nonanastomotic pseudoaneurysm with complete disruption of an expanded polytetrafluoroethylene axillofemoral bypass graft. Ann Vasc Surg. 2012;26(3):422.e9-422.12. PubMed

18. Selvarasu NKC, Tafti DK, Vlachos PP. Hydrodynamic effects of compliance mismatch in stented arteries. J Biomech Eng. 2011;133(2):021008.

19. Gasser TC, Ogden RW, Holzapfel GA. Hyperelastic modelling of arterial layers with distributed collagen fibre orientations. J R Soc Interface. 2006;3(6):15-35.

20. Verbeke G, Molenberghs G. Linear Mixed odels for Longitudinal Data. New York: Springer; 2000. 